\title{
Feasibility of using stereotactic body radiation therapy for unresectable soft tissue tumors of the trunk
}

\author{
Eun Kyung Paik ${ }^{1}$, Mi-Sook Kim ${ }^{1}$, Chul-Koo Cho ${ }^{1}$, Hyung Jun Yoo ${ }^{1}$, Won Il Jang ${ }^{1}$, \\ Young-Seok Seo ${ }^{2}$, Sung-Ho $\mathrm{Jin}^{3}$, Dae Geun Jeon ${ }^{4}$ and Dong Han Lee ${ }^{5}$ \\ ${ }^{1}$ Department of Radiation Oncology, Korea Institute of Radiological and Medical Sciences, Seoul, Korea \\ ${ }^{2}$ Department of Radiation Oncology, Seoul National University Hospital, Seoul, Korea \\ ${ }^{3}$ Department of Surgery, Korea Institute of Radiological and Medical Sciences, Seoul, Korea \\ ${ }^{4}$ Department of Orthopedic Surgery, Korea Institute of Radiological and Medical Sciences, Seoul, Korea \\ ${ }^{5}$ CyberKnife Center, Korea Institute of Radiological and Medical Sciences, Seoul, Korea \\ Correspondence to: Mi-Sook Kim, email: mskim@kirams.re.kr \\ Keywords: soft tissue tumor; sarcoma; stereotactic body radiation therapy; unresectable; trunk \\ Received: September 07, $2017 \quad$ Accepted: May 17, $2018 \quad$ Published: June 12, 2018 \\ Copyright: Paik et al. This is an open-access article distributed under the terms of the Creative Commons Attribution License 3.0 \\ ( $C$ C BY 3.0), which permits unrestricted use, distribution, and reproduction in any medium, provided the original author and source \\ are credited.
}

\section{ABSTRACT}

Purpose: To evaluate the feasibility of stereotactic body radiation therapy (SBRT) for unresectable soft tissue tumors of the trunk.

Materials and Methods: Between January 2002 and December 2008, 23 patients with 36 lesions of soft tissue tumors, which were located in the trunk and not suitable for resection, underwent SBRT. Among the 36 lesions, 31 were malignant and 5 were benign. The median tumor volume was $24 \mathrm{~cm}^{3}$ (range, $2.6-213 \mathrm{~cm}_{3}$ ). SBRT doses ranged from 20 to $48 \mathrm{~Gy}$ in 1-5 fractions.

Results: With a median follow-up of 73 months, the overall survival (OS) and local control (LC) rates at 5 years were $39 \%$ and $52 \%$, respectively. For malignant tumors, the OS and LC rates at 5 years were $28 \%$ and $47 \%$, respectively. For benign tumors, the OS and LC rates at 5 years were $80 \%$ and $100 \%$, respectively. There was no acute toxicity of grade $\geq 3$. One case of grade 3 late skin toxicity was reported 10 months after SBRT.

Conclusion: SBRT may be an effective and safe treatment modality for the local control of unresectable soft tissue tumors of the trunk including tumors of a benign nature.

\section{INTRODUCTION}

Soft tissue sarcomas (STSs) are rare tumors arising from connective tissues that can occur in any anatomical site. They represent about $1 \%$ of cancers diagnosed in the US with an annual incidence of about 12,000 cases [1]. The extremities are the most common sites of presentation, accounting for approximately $60 \%$ of cases, followed by trunk (15-20\%), retroperitoneum (10-15\%), and the head and neck (8\%) [2]. The aim of the treatment of STS is to achieve complete eradication of the tumor with optimal function preservation and minimal treatment-related toxicities. Several randomized trials have been performed and have established conservation surgery combined with radiation therapy (RT) as the standard management for most STSs of the extremities and trunk [3-5]. However, the cumulative probability of local recurrence at 5 years in extremity STS, as reported in large series, remains as high as $20 \%$ [3-6].

Due to their deep location, presentation of soft tissue tumors in the trunk is often delayed until the tumor becomes very large. Analysis of the Surveillance Epidemiology and End Results (SEER) database showed that the median tumor size in the case of retroperitoneal 
tumors is $15.5 \mathrm{~cm}$ (range, $0.5-99.5 \mathrm{~cm}$ ) [7]. Therefore, it is often difficult to achieve complete resection with an optimal margin. As a result, following frequent local recurrences and repeated operations, the tumor eventually becomes inoperable. For inoperable STS, palliative chemotherapy has been the long-standing treatment option. In certain cases, such as for well-localized tumors in the extremities, the overall survival (OS) rate at 2 years with palliative chemotherapy is reported to be up to $28-31 \%$ [8]. Despite this, only a few patients achieve an objective response. In situations where the effect of chemotherapy for STS is uncertain, RT is the choice for local treatment. However, for the same reason of difficulty with surgery, sufficient doses of radiation may not be administered for STSs of the trunk, resulting in radiation treatment administered mostly with a palliative aim, such as pain relief.

Owing to remarkable advances in technology for tumor imaging and radiation delivery systems, it has become possible in recent years to treat a variety of cancers with stereotactic body radiation therapy (SBRT) with highdose per fraction. One of the advantages of treating tumors with SBRT over the conventional external beam radiation therapy (EBRT) is that SBRT precisely irradiate tumors while allowing tight margin of surrounding normal tissues. Furthermore, because the $\alpha / \beta$ ratio of STS is known to be relatively low with a range of 1.4-5.4 [9], the therapeutic gain with SBRT in 1-5 fractions may be greater than that with the conventional multi-fractionated radiotherapy. Despite these theoretical advantages, there has been little definitive clinical investigation. The purpose of this study was to evaluate the feasibility and safety of SBRT for unresectable soft tissue tumors of the trunk, including malignant tumors and pathologically benign tumors exhibiting malignant behavior.

\section{RESULTS}

\section{Treatment outcomes}

The treatment outcomes of SBRT to 23 patients with 36 lesions of unresectable soft tissue tumors located in the trunk were analyzed. The baseline patient and tumor characteristics are shown in Table 1. With a median follow-up of 73 months for patients alive at the last followup, the local control (LC) rate at 5 years was 52\%, and the OS rate at 5 years was 39\%. For malignant tumors (18 patients with 31 lesions), the OS and LC rates at 5 years were $28 \%$ and $47 \%$, respectively (Figure 1A). For benign tumors (5 patients with 5 lesions), the OS and LC rates at 5 years were $100 \%$ and $80 \%$, respectively (Figure 1B).

In order to investigate the effect of prognostic factors on LC and OS rates, univariate analysis was performed. Only tumor characteristics of a benign or malignant nature had a significant impact on OS $(P=0.033)$, and no significant prognostic factor was associated with LC.
The 5 benign tumors were treated with SBRT because they were unresectable due to their location and previous operation histories (Table 2). Patient 1 had undergone 7 previous surgeries for different lesions, and the treated tumor in the buttock area recurred 8 months after surgery. The size of the mass was increasing and the patient was complaining symptoms of pain. Patient 2 had a recurrent tumor in the paraspinal (sacral) area 1 year after surgery. The tumor showed a rapid growing pattern, and the patient was referred for RT hoping to slow down the growth or to reduce the tumor volume. Patient 3 had undergone 4 previous surgeries for different lesions, and the treated tumor in the chest wall recurred 9 years after surgery. The patient refused surgery. Patient 4 had a tumor in the paraspinal (lumbar) area, which was followed up for 15 years before radiating pain developed, and was treated with SBRT. Patient 5 had a tumor in the paraspinal (lumbar) area. Although it was asymptomatic, the size of the tumor continuously increased during 3 years of follow-up. One patient (patient 5) died during follow-up 20 months after SBRT due to hepatocellular carcinoma. All of the lesions treated with SBRT remained stable, symptoms were relieved, and none of the lesions needed further treatment. Except for the patient who died 20 months after SBRT, long-term LC of 73-128 months was achieved.

Five re-irradiated malignant cases were also included in this study (Table 3). Four patients had undergone previous operations. Among them 1 patient received preoperative RT and 3 patients received postoperative RT. One patient without previous surgery had received previous SBRT of 20 Gy in 1 fraction. All 5 patients showed local recurrences and distant metastases during follow-up. Local recurrence occurred in all lesions within 2 years, and all patients expired within 3 years. The median LC for the re-irradiated lesions was 14 months (range, 4-23 months). The median OS was 22 months (range, 12-28 months).

\section{Toxicity}

Toxicities were investigated and graded according to the Common Terminology Criteria for Adverse Events (CTCAE) v4.0. There were no severe acute gastrointestinal, genitourinary, or skin toxicities of grade $\geq 3$. Grade 3 late skin toxicity was observed in 1 reirradiated case 10 months after SBRT treatment (Table 3). The tumor was treated with SBRT with a dose of 30 Gy in 3 fractions delivered in 3 consecutive days. The patient had undergone several previous surgeries due to repeated recurrences, and was previously treated with 60 Gy in 30 fractions of postoperative RT 64 months before the retreatment with SBRT. The patient experienced wet desquamation of the skin from 1 month after SBRT. Ten months after the SBRT treatment, the patient was hospitalized for antibiotic treatment due to radiation 
Table 1: Baseline patient and tumor characteristics

\begin{tabular}{|c|c|c|c|}
\hline Characteristics & Range (median) & No. of patients $(\%)(N=23)$ & No. of lesions $(\%)(N=36)$ \\
\hline \multicolumn{4}{|l|}{ Gender } \\
\hline Male & & $14(61)$ & \\
\hline Female & & $9(39)$ & \\
\hline Age & $23-72$ years $(41)$ & & \\
\hline \multicolumn{4}{|l|}{ Location } \\
\hline Chest & & & $15(42)$ \\
\hline Abdomen & & & $8(22)$ \\
\hline Pelvis & & & $13(36)$ \\
\hline \multicolumn{4}{|l|}{ Presentation } \\
\hline Primary & & $3(13)$ & $3(8)$ \\
\hline Recurrent & & $20(87)$ & $33(92)$ \\
\hline \multicolumn{4}{|l|}{ Tumor characteristics } \\
\hline Benign & & $5(22)$ & $5(14)$ \\
\hline Malignant & & $18(78)$ & $31(86)$ \\
\hline \multicolumn{4}{|l|}{ Histology } \\
\hline $\begin{array}{l}\text { Undifferentiated } \\
\text { pleomorphic sarcoma }\end{array}$ & & $5(22)$ & $12(33)$ \\
\hline Synovial sarcoma & & $4(17)$ & 7 (19) \\
\hline Epithelioid sarcoma & & $2(9)$ & $3(8)$ \\
\hline Hemangiopericytoma & & $1(4)$ & $3(8)$ \\
\hline Others & & $11(48)$ & $11(29)$ \\
\hline Tumor volume & $2.6-213 \mathrm{~cm}^{3}(24)$ & & \\
\hline
\end{tabular}

necrosis and infections of the skin in the treated area. No radiation-induced secondary malignancies were observed.

\section{DISCUSSION}

In the present study, the SBRT of unresectable STS of the trunk with prescribed doses of $20-48$ Gy in 1-5 fractions resulted in an LC rate of $52 \%$ and an OS rate

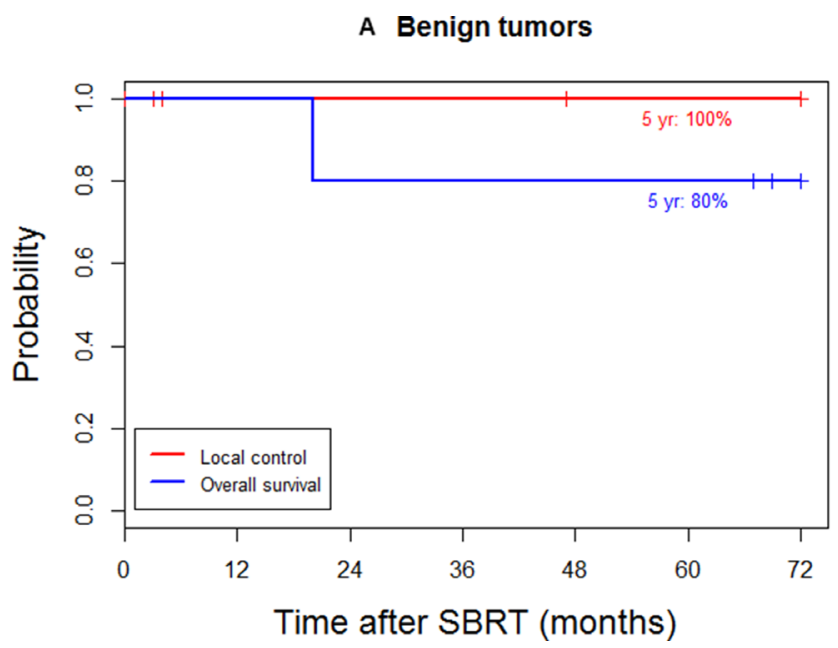

of $39 \%$ at 5 years. The specific dose prescriptions and biologically equivalent doses (BEDs) for malignant tumors initially treated with SBRT are shown in Table 4. Due to the location of the tumor and nearby organs at risk, various dose fractionations were applied. When it is assumed that the $\alpha / \beta$ ratio of STS is $4[10,11]$, the total dose converted to equivalent doses in 2 Gy-fractions (EQD2) was 72-240 $\mathrm{Gy}_{4}$ (median $144 \mathrm{~Gy}_{4}$ ). For cases of $\mathrm{R} 1$ resection margins

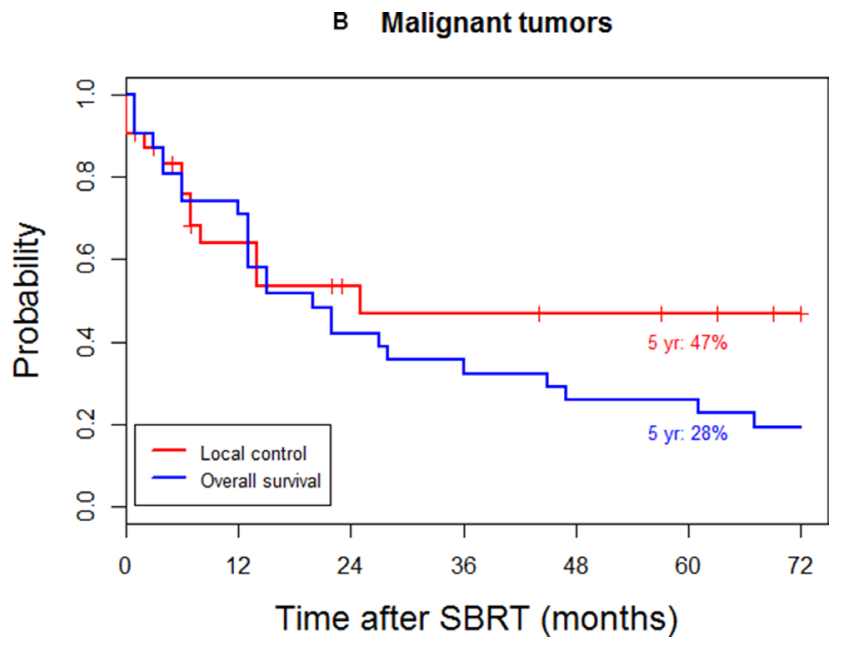

Figure 1: Local control and overall survival for (A) benign and (B) malignant tumors. 
Table 2: Characteristics of benign soft tissue tumors

\begin{tabular}{lccccccc}
\hline $\begin{array}{l}\text { Patient } \\
\text { No. }\end{array}$ & Sex/Age & Histology & Location & Volume $\left(\mathbf{c m}^{\mathbf{3}}\right)$ & Dose $(\mathbf{G y} / \mathbf{f x})$ & $\mathbf{B E D}\left(\mathbf{G y} \mathbf{y}_{\mathbf{3}}\right)$ & $\mathbf{F} / \mathbf{U}^{\text {a) }}(\mathbf{M})$ \\
\hline 1 & $\mathrm{~F} / 50$ & Fibromatosis & Buttock & 38.4 & $33 / 3$ & 92 & 128 \\
2 & $\mathrm{M} / 32$ & Giant cell tumor & Paraspinal & 143.8 & $42 / 3$ & 143 & 102 \\
3 & $\mathrm{M} / 32$ & Neurofibroma & Chest wall & 9.5 & $24 / 3$ & 53 & 86 \\
4 & $\mathrm{M} / 37$ & Hemangioblastoma & Paraspinal & 11.2 & $24 / 3$ & 53 & 73 \\
5 & $\mathrm{M} / 56$ & Neurogenic tumor & Paraspinal & 33.7 & $27 / 3$ & 65 & $20^{\text {b) }}$ \\
\hline
\end{tabular}

Abbreviations: BED, biologically equivalent dose; F/U, follow-up after SBRT; M, months

a)All patients achieved local control during follow-up.

b)Patient 5 died from hepatocellular carcinoma at 20 months after SBRT.

Table 3: Characteristics of re-irradiated cases

\begin{tabular}{|c|c|c|c|c|c|c|c|c|c|}
\hline Sex/Age & Histology & Location & $\begin{array}{l}\text { Volume } \\
\left(\mathrm{cm}^{3}\right)\end{array}$ & $\begin{array}{l}\text { Previous } \\
\text { RT dose } \\
\text { (Gy/fx) }\end{array}$ & $\begin{array}{l}\text { SBRT } \\
\text { dose } \\
(\mathbf{G y} / \mathbf{f x})\end{array}$ & $\begin{array}{l}\text { Durationa) }^{\text {a }} \\
\text { (M) }\end{array}$ & $\begin{array}{l}\mathbf{O S}^{\mathbf{b})} \\
(\mathrm{M})\end{array}$ & $\begin{array}{l}\mathbf{L C} \mathbf{C}^{\mathbf{c}} \\
(\mathbf{M})\end{array}$ & $\begin{array}{l}\text { Toxicity } \\
\text { y } \geq \text { Gr } 3\end{array}$ \\
\hline $\mathrm{F} / 31$ & $\begin{array}{l}\text { Synovial } \\
\text { sarcoma }\end{array}$ & Pelvis & 33.7 & $30 / 10$ & $24 / 3$ & 1 & 28 & 14 & None \\
\hline $\mathrm{F} / 52$ & $\begin{array}{c}\text { Malignant } \\
\text { schwannoma }\end{array}$ & Back & 213.8 & $60 / 30$ & $30 / 3$ & 64 & 27 & 23 & Skin $^{\text {d) }}$ \\
\hline $\mathrm{M} / 63$ & $\begin{array}{l}\text { Undifferentiated } \\
\text { pleomorphic } \\
\text { sarcoma }\end{array}$ & Pelvis & 9.4 & $60 / 30$ & $40 / 5$ & 25 & 22 & 14 & None \\
\hline $\mathrm{F} / 34$ & $\begin{array}{l}\text { Myofibroblastic } \\
\text { sarcoma }\end{array}$ & Buttock & 124.6 & $45 / 15$ & $39 / 3$ & 19 & 20 & 4 & None \\
\hline $\mathrm{M} / 31$ & $\begin{array}{l}\text { Synovial } \\
\text { sarcoma }\end{array}$ & Paraspinal & 59.3 & $20 / 1$ & $30 / 3$ & 20 & 12 & 7 & None \\
\hline
\end{tabular}

Abbreviations: RT, radiation therapy; SBRT, stereotactic body radiation therapy; OS, overall survival; M, months; LC, local control; Gr, grade.

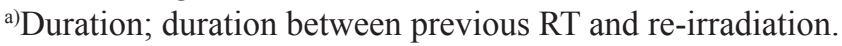

b)All patients expired within 3 years after SBRT.

${ }^{c}$ All lesions recurred within 2 years after SBRT.

${ }^{d}$ Grade 3 skin toxicity occurred at $10 \mathrm{M}$ after SBRT.

(microscopic positive margins), R2 resection margins (macroscopic positive margins), and unresectable tumors treated with conventional RT, 5-year LC rates are 39-52\% [12-14]. In a retrospective study by Youssef et al. [12], tumors in the retroperitoneum and deep trunk were treated with surgery plus conventional EBRT, with or without brachytherapy. RT doses were EBRT 52.2 Gy or EBRT 42 Gy plus brachytherapy $16 \mathrm{~Gy}$, and showed a 5-year OS rate of $48 \%$ and a 5 -year LC rate of $52 \%$. In a study by Kepka et al. [13], tumors in the extremities, retroperitoneum, head and neck, and the truncal wall were treated with conventional EBRT, with or without chemotherapy. With a median RT dose of $64 \mathrm{~Gy}$ (range, 25-87.5 Gy), they showed a 5 -year OS rate of $35 \%$ and a 5 -year LC rate of $45 \%$. In a study by Feng et al. [14], tumors in the retroperitoneum, pelvis, and deep trunk were treated with EBRT, with or without surgery. The median RT dose was 56.4 Gy (range, $7-73 \mathrm{~Gy})$, and the outcome was a 5-year OS rate of $12 \%$ and a 5 -year LC rate of 39\%. The results of SBRT for sarcomas of spine have been reported [15-17], but to our knowledge, our present study is the first one to investigate the SBRT for the sarcomas of the trunk. The overall results of our study were comparable to the above mentioned previously reported results of conventional fractionated radiotherapy [12-14]. As shown in Table 4, the BED values calculate using $\alpha / \beta=4$ and 10 varied considerably. However, considering the high dose when converted to BED with $\alpha / \beta$ $=4$, an LC of approximately $50 \%$ seems low. Initially, when we decided on the SBRT dose fractionations, we converted the doses using $\alpha / \beta=10$. As a result, the SBRT doses we used are slightly higher than the conventional doses used in the literature. Therefore $\alpha / \beta$ may not be as low as 4 , as suggested from the literature. However, in light of the increasing evidence that the linear-quadratic model is not valid for irradiation with a large dose/fraction, there are limitations in interpreting the results using BEDs. 
Table 4: Details of SBRT doses of initially treated malignant STSs

\begin{tabular}{lcccc}
\hline Fractions & Total dose $\mathbf{( G y )}$ & No. of patients treated & BED $\left(\mathbf{G y}_{\mathbf{4}}\right)$ & $\mathbf{B E D}\left(\mathbf{G \mathbf { y } _ { \mathbf { 1 0 } }}\right)$ \\
\hline 1 & 20 & 3 & 120 & 60 \\
& 24 & 1 & 168 & 82 \\
26 & 3 & 195 & 94 \\
26 & 1 & 111 & 60 \\
& 28 & 1 & 126 & 67 \\
& 2 & 160 & 83 \\
3 & 2 & 2 & 88 & 51 \\
& 27 & 1 & 124 & 69 \\
& 33 & 4 & 144 & 79 \\
& 36 & 5 & 166 & 90 \\
& 39 & 2 & 214 & 113 \\
& 45 & 1 & 240 & 125 \\
\hline
\end{tabular}

Abbreviations: SBRT, stereotactic body radiation therapy; STS, soft tissue sarcoma; BED, biologically equivalent dose.

There were 5 cases of benign soft tissue tumors included in our study. Although pathologically benign, some of the tumors behaved like malignant tumors from the point of view of local invasiveness or multiple recurrences after surgery. Benign tumors fall under the radiobiological category of late-responding tissues, and are assumed to have a low $\alpha / \beta$ ratio [18]. Therefore, hypofractionated radiotherapy may be more effective than conventional fractionated radiotherapy for benign tumors. The benign soft tissue tumors of 5 patients were unresectable owing to the location of the tumors and previous operation histories. Our study showed excellent LC and OS rates for benign tumors treated with SBRT with a dose of 24-42 Gy in 3 fractions delivered on consecutive days. If converted into EQD2 with $\alpha / \beta=3$, the doses were $53-143 \mathrm{~Gy}_{3}$. All of the treated lesions remained stable after treatment with SBRT, and none of the lesions needed further treatment. One patient died during follow-up due to hepatocellular carcinoma, but the treated lesion was stable 20 months after SBRT. None of the patients experienced severe complications of grade $\geq 3$. Therefore, SBRT might be a promising treatment modality for unresectable benign soft tissue tumors in patients with a history of several operations and those presenting with uncontrolled pain. However, the number of patients in our study is small, and further evidence needs to be accumulated.

In our study, 5 patients were re-irradiated for recurrence with SBRT. Most of the patients had undergone several previous surgeries due to multiple recurrences. Studies in which patients were retreated with EBRT show serious complication rates of $42-60 \%$. Since SBRT allows delivery of high doses of radiation to limited volumes of tissue whilst sparing the surrounding normal tissues, SBRT may be applied for re-irradiation. In similar rationale, brachytherapy has been used for recurrent STS cases in the extremities after previous radiation treatment. However, there may be some limitations for applying brachytherapy due to large tumor volumes, restrictions in catheter placement due to normal tissue anatomy, and risk of radiation injury to normal tissues in direct contact with the catheters [19]. Previous studies of brachytherapy for re-irradiation shows results derived from diverse tumor locations and tumor sizes. Therefore, direct comparison of our results with the results from other studies would be difficult. However, it would be useful to look into these studies for some insights regarding complications. In a study of brachytherapy from Memorial Sloan-Kettering Cancer Center, the 5 -year OS and LC rates were $85 \%$ and $68 \%$, respectively [5]. However, a $12.5 \%$ rate of serious complications was observed with a median follow-up of 3 years. A study from the MD Anderson Cancer Center suggested that re-irradiation does not clearly improve outcome after surgical excision alone, but in fact increases complications [20]. Using brachytherapy, patients were treated with 45-50 Gy. The 5-year LC and 5-year diseasespecific survival rates were $51 \%$ and $65 \%$, respectively, but serious complication rates were as high as $75 \%$. In a study from the University of Florida, patients were retreated with radiation, including brachytherapy, for recurrent STSs [21]. The median RT dose was 50.4 Gy (range, 38.0-66.0) at presentation, and 57.6 Gy (43.2-66.0) at recurrence. For retreatment, the 5-year OS and LC rates were $40 \%$ and $18 \%$, respectively, but there was a $50 \%$ incidence of serious complications. In our study, 1 patient showed grade 3 skin toxicity 10 months after SBRT. The patient was previously treated with $60 \mathrm{~Gy}$ in 30 fractions 64 months before being retreated with SBRT of $30 \mathrm{~Gy}$ in 3 fractions. However, the patient had also undergone several previous surgeries on the treated site, and the toxicity may have resulted from combined treatments. Therefore, even when it is possible to treat with a palliative aim, care must be taken not to cause complications.

In conclusion, the present study demonstrated that SBRT is an effective and safe treatment modality for unresectable soft tissue tumors of the trunk, with a low risk 
of severe toxicity. SBRT may have role in the management of benign soft tissue tumors, showing malignant behavior by providing satisfactory progression-free survival without the need for further treatment. However, for re-irradiation cases, more evidence needs to be accumulated before drawing any conclusions.

\section{MATERIALS AND METHODS}

\section{Patients}

Twenty-three patients with unresectable soft tissue tumors located in the trunk were treated with SBRT using CyberKnife (Accuray Inc., Sunnyvale, CA, USA) during January 2002 to December 2008 at our institute. The medical records of a total of 23 patients with 36 lesions located in the trunk that were unsuitable for resection were retrospectively reviewed. Patients who were treated with conventional EBRT followed by SBRT boost were excluded. This study was approved by the Institutional Review Board of our institute.

Fourteen patients were male and 9 were female. Ages ranged from 23 to 72 years with a median of 41 years. The size of the tumor ranged from 2.6 to $213 \mathrm{~cm}^{3}$, with a median of $24 \mathrm{~cm}^{3}$. The locations of the lesions by anatomical site were diverse and were as follows. Fifteen lesions were in the chest, including 4 lung lesions, 3 chest wall lesions, 3 axilla lesions, 3 paraspinal lesions, 1 back lesion, and 1 paracardiac lesion. Eight lesions were in the abdomen, including 5 paraspinal lesions, 1 paraaortic lesion, 1 anterior abdominal wall lesion, and 1 liver lesion. Thirteen lesions were in the pelvis, including 4 intrapelvic lesions, 3 buttock lesions, 2 parasacral lesions, 2 inguinal lesions, 1 perineal lesion, and 1 perianal lesion. Three were primary cases and 33 were recurrent cases. Five re-irradiated cases were also included. Five had benign tumor characteristics and 31 were malignant. The histology of the tumors was heterogeneous, including 12 undifferentiated pleomorphic sarcoma, 7 synovial sarcomas, 3 epithelioid sarcomas, 3 hemangiopericytomas, 1 liposarcoma, 1 giant cell tumor, 1 fibromatosis, 1 neurofibroma, 1 hemangioblastoma, 1 malignant schwannoma, 1 rhabdomyosarcoma, 1 follicular dendritic cell sarcoma, 1 myofibroblastic sarcoma, 1 neurogenic tumor, and 1 malignant peripheral nerve sheath tumor.

\section{Stereotactic body radiation therapy}

Patients were treated with SBRT using the CyberKnife system. The gross tumor identified on the simulation computed tomography (CT) scan was defined as the gross tumor volume (GTV). A margin of 0-4 mm was added to the GTV for the planning target volume (PTV). SBRT doses were prescribed at an isodose line (64-83\% of the maximum dose) that covered at least $97 \%$ of the PTV. A total dose of 20-48 Gy (median 32 Gy) in 1-5 fractions was prescribed. The overall treatment time for those except single treatment cases were 2-11 days. Most of the patients received treatment on consecutive days excluding weekends. For the patients who did not receive consecutive treatments (4 patients, 5 lesions), 1 patient received 2 fractions of treatment 11 days apart due to nausea and vomiting after the first treatment, another patient had 2 lesions treated with 3 fractions each alternatively for a total of 7 days, and the other 2 patients received 3 fractions of treatment each with at least 48 hours apart. The BED, when calculated with $\alpha / \beta=10$, ranged from $43 \mathrm{~Gy}_{10}$ to $125 \mathrm{~Gy}_{10}$, with a median BED of $79 \mathrm{~Gy}_{10}$, and with $\alpha / \beta=4$, BED ranged from $72 \mathrm{~Gy}_{4}$ to $240 \mathrm{~Gy}_{4}$, with a median BED of $144 \mathrm{~Gy}_{4}$. If converted into EQD2, the prescribed dose was 36-104 $\mathrm{Gy}_{10}$ with a median EQD2 of $66 \mathrm{~Gy}_{10}$ for $\alpha / \beta=10$, and for $\alpha / \beta=4$, the prescribed dose was 48-160 $\mathrm{Gy}_{4}$, with a median EQD2 of $96 \mathrm{~Gy}_{4}$.

\section{Statistical analysis}

LC and OS rates for all patients were calculated from the date of the SBRT using the Kaplan-Meier method. Univariate analysis was performed using the logrank test to identify significant prognostic factors for LC and OS. For all analyses, two-sided tests of significance were used with $P$ values $<0.05$ considered significant. All statistical analyses were performed using the Statistical Package for the Social Sciences (version 14.0; SPSS, Inc. Chicago, IL, USA).

\section{Abbreviations}

STS: soft tissue sarcoma; RT: radiation therapy; OS: overall survival; SBRT: stereotactic body radiation therapy; EBRT: external beam radiation therapy; LC: local control; BED: biologically equivalent dose; EQD2: equivalent doses in 2 Gy-fractions; GTV: gross tumor volume; PTV: planning target volume.

\section{Author contributions}

EKP, MSK: study concept and design, acquisition of data, analysis and interpretation of data, drafting of the initial manuscript. CHK, HYJ, WIJ, YSS, SHJ, DGJ, DHL: acquisition of data, analysis and interpretation of data.

\section{ACKNOWLEDGMENTS AND FUNDING}

This study was supported by a grant of the Korea Institute of Radiological \& Medical Sciences (KIRAMS), funded by Ministry of Science and ICT (MSIT), Republic of Korea. (No.50543-2018).

\section{CONFLICTS OF INTEREST}

The authors declare that they have no conflicts of interest. 


\section{REFERENCES}

1. Siegel RL, Miller KD, Jemal A. Cancer statistics, 2017. CA Cancer J Clin. 2017; 67:7-30.

2. Lawrence W Jr, Donegan WL, Natarajan N, Mettlin C, Beart R, Winchester D. Adult soft tissue sarcomas. A pattern of care survey of the American College of Surgeons. Ann Surg. 1987; 205:349-59.

3. Rosenberg SA, Tepper J, Glatstein E, Costa J, Baker A, Brennan M, DeMoss EV, Seipp C, Sindelar WF, Sugarbaker P, Wesley R. The treatment of soft-tissue sarcomas of the extremities: prospective randomized evaluations of (1) limb-sparing surgery plus radiation therapy compared with amputation and (2) the role of adjuvant chemotherapy. Ann Surg. 1982; 196:305-15.

4. Yang JC, Chang AE, Baker AR, Sindelar WF, Danforth DN, Topalian SL, DeLaney T, Glatstein E, Steinberg SM, Merino MJ, Rosenberg SA. Randomized prospective study of the benefit of adjuvant radiation therapy in the treatment of soft tissue sarcomas of the extremity. J Clin Oncol. 1998; 16:197-203.

5. Pisters PW, Harrison LB, Leung DH, Woodruff JM, Casper ES, Brennan MF. Long-term results of a prospective randomized trial of adjuvant brachytherapy in soft tissue sarcoma. J Clin Oncol. 1996; 14:859-68.

6. Mundt AJ, Awan A, Sibley GS, Simon M, Rubin SJ, Samuels B, Wong W, Beckett M, Vijayakumar S, Weichselbaum RR. Conservative surgery and adjuvant radiation therapy in the management of adult soft tissue sarcoma of the extremities: clinical and radiobiological results. Int J Radiat Oncol Biol Phys. 1995; 32:977-85.

7. Tseng WH, Martinez SR, Do L, Tamurian RM, Borys D, Canter RJ. Lack of survival benefit following adjuvant radiation in patients with retroperitoneal sarcoma: a SEER analysis. J Surg Res. 2011; 168:e173-80.

8. Judson I, Verweij J, Gelderblom H, Hartmann JT, Schoffski P, Blay JY, Kerst JM, Sufliarsky J, Whelan J, Hohenberger P, Krarup-Hansen A, Alcindor T, Marreaud S, et al. Doxorubicin alone versus intensified doxorubicin plus ifosfamide for first-line treatment of advanced or metastatic soft-tissue sarcoma: a randomised controlled phase 3 trial. Lancet Oncol. 2014; 15:415-23.

9. Thames HD, Suit HD. Tumor radioresponsiveness versus fractionation sensitivity. Int J Radiat Oncol Biol Phys. 1986; 12:687-91.

10. Soyfer V, Corn BW, Kollender Y, Tempelhoff H, Meller I, Merimsky O. Radiation therapy for palliation of sarcoma metastases: a unique and uniform hypofractionation experience. Sarcoma. 2010; 2010:927972.
11. Cuneo KC, Riedel RF, Dodd LG, Harpole DH Jr, Kirsch DG. Pathologic complete response of a malignant peripheral nerve sheath tumor in the lung treated with neoadjuvant Ifosfamide and radiation therapy. J Clin Oncol. 2012; 30:e291-3.

12. Youssef E, Fontanesi J, Mott M, Kraut M, Lucas D, Mekhael H, Ben-Josef E. Long-term outcome of combined modality therapy in retroperitoneal and deep-trunk softtissue sarcoma: analysis of prognostic factors. Int J Radiat Oncol Biol Phys. 2002; 54:514-9.

13. Kepka L, DeLaney TF, Suit HD, Goldberg SI. Results of radiation therapy for unresected soft-tissue sarcomas. Int $\mathrm{J}$ Radiat Oncol Biol Phys. 2005; 63:852-9.

14. Feng M, Murphy J, Griffith KA, Baker LH, Sondak VK, Lucas DR, McGinn CJ, Ray ME. Long-term outcomes after radiotherapy for retroperitoneal and deep truncal sarcoma. Int J Radiat Oncol Biol Phys. 2007; 69:103-10.

15. Chang UK, Cho WI, Lee DH, Kim MS, Cho CK, Lee SY, Jeon DG. Stereotactic radiosurgery for primary and metastatic sarcomas involving the spine. J Neurooncol. 2012; 107:551-7.

16. Leeman JE, Bilsky M, Laufer I, Folkert MR, Taunk NK, Osborne JR, Arevalo-Perez J, Zatcky J, Alektiar KM, Yamada Y, Spratt DE. Stereotactic body radiotherapy for metastatic spinal sarcoma: a detailed patterns-of-failure study. J Neurosurg Spine. 2016; 25:52-8.

17. Miller JA, Balagamwala EH, Angelov L, Suh JH, Djemil T, Magnelli A, Qi P, Zhuang T, Godley A, Chao ST. Stereotactic radiosurgery for the treatment of primary and metastatic spinal sarcomas. Technol Cancer Res Treat. 2017; 16:276-84.

18. Hall EJ, Brenner DJ. The radiobiology of radiosurgery: rationale for different treatment regimes for AVMs and malignancies. Int J Radiat Oncol Biol Phys. 1993; 25:381-5.

19. Holloway CL, Delaney TF, Alektiar KM, Devlin PM, O'Farrell DA, Demanes DJ. American Brachytherapy Society (ABS) consensus statement for sarcoma brachytherapy. Brachytherapy. 2013; 12:179-90.

20. Torres MA, Ballo MT, Butler CE, Feig BW, Cormier JN, Lewis VO, Pollock RE, Pisters PW, Zagars GK. Management of locally recurrent soft-tissue sarcoma after prior surgery and radiation therapy. Int J Radiat Oncol Biol Phys. 2007; 67:1124-9.

21. Indelicato DJ, Meadows $\mathrm{K}$, Gibbs CP Jr, Morris CG, Scarborough MT, Zlotecki RA. Effectiveness and morbidity associated with reirradiation in conservative salvage management of recurrent soft-tissue sarcoma. Int J Radiat Oncol Biol Phys. 2009; 73:267-72. 